\title{
Biohydrogen-generating capability of microflora after prolonged substrate limitation
}

\begin{abstract}
The ability of heat-treated cow dung microflora to generate biohydrogen was investigated in an anaerobic digester after subjecting it to prolonged substrate limitation. Cow dung microflora exhibited hydrogen generating capability in our earlier studies when fed with substrate like palm oil mill effluent and jackfruit peel waste. Performance of hydrogen generating microflora was monitored by subjecting it to substrate limiting condition for a period of 91 days. Results showed that a minimum hydrogen content of $0.5 \%$ was observed on the 75th day of substrate limiting period. Thereafter, the composition of biogas could not be measured as the generated volume was low. Recovery of anaerobic digester was attempted by feeding a mixture of brewery waste and anaerobic agar medium under a continuous mode for a hydraulic retention time of 4 days at an influent $\mathrm{pH}$ value of $5.1 \pm 0.2$. During the recovery phase of the digester, the hydrogen content of the anaerobic digester was found to be 18 and 50\% during the 30th and 77th day of feeding operation. The foregoing studies proved that the spore enriched cow dung microflora can maintain their viability during substrate limiting condition.
\end{abstract}

Keyword: Anaerobic contact filter; Beer brewery wastewater; Biohydrogen; Cow dung; Substrate limiting condition 